\title{
Nanoscale Observation of Intact Biological Specimens in Water with High-contrast Imaging by Scanning Electron Assisted Dielectric-impedance Microscopy
}

\author{
T. Ogura ${ }^{1}$ and T. Okada ${ }^{1}$ \\ 1. Biomedical Research Institute, National Institute of Advanced Industrial Science and Technology \\ (AIST), Central 2, Umezono, Tsukuba, Ibaraki, Japan
}

Scanning electron microscopy (SEM) has been widely used to analyse biological specimens. However, SEM observations of these specimens under high vacuum conditions require specific sample preparation protocols involving glutaraldehyde fixation, negative staining, cryo-techniques and metal coating to avoid electrical radiation damage. These preparations also have positive effects in terms of contrast enhancement. To date, atmospheric and/or wet biological specimens have been examined using atmospheric holders, but they undergo heavy radiation damage caused by electron beam (EB).

We recently developed a new imaging technology called a scanning-electron assisted dielectricimpedance microscopy (SE-ADM) system based on SEM [1-2]. Our system provides high-contrast imaging of the intact biological specimens in water introduced in an atmospheric sample holder comprising two silicon nitride ( $\mathrm{SiN}$ ) films (Fig. 1a). The upper SiN film, which is coated with tungsten (W), is irradiated using a focused scanning EB. Irradiated electrons are strongly scattered and absorbed in the $15 \mathrm{~nm}$-thick $\mathrm{W}$ layer resulting in a negative electric-field potential at the irradiated position. This negative potential transmitted to the bottom SiN film through the biological specimens in water (Fig. 1b) [1]. In this method, the biological samples are not directly exposed to the EB, which prevents electron radiation damage [1].

The intact bacteria (Rhodobacter capsulatus) immersed in water were examined using the highresolution SE-ADM system at 10,000× magnification, 3.6-kV EB acceleration [2]. The intact bacteria were visible with clear-cut white contrast; the inner structure exhibited complex undulation (Fig. 2a). We also used the new method to analyse unstained and unfixed IgM antibody molecules in water. The IgM antibody has molecular weight of $900 \mathrm{kDa}$ and consists of five IgG antibodies. We examined intact IgM protein in water at 50,000× magnification and 4-kV EB acceleration (Fig. 2b). Several small particles of approximately 50-nm diameter were dispersed throughout the whole visual field. Fig. 2c shows three individual IgM molecules indicated in Fig. $2 \mathrm{~b}$ with white arrows, and the pseudo-colour maps are shown in Fig. 2d. Individual IgM molecules are visible as slightly star shaped with a dome-like centre. Further, we demonstrate the nanoscale observation of living mammalian cells using SE-ADM system with a culture dish holder [3]. Our system clearly visualised unstained and unfixed cancer cells in medium (Fig. 3). Figure 3a clearly shows intracellular structures of the nucleus and endoplasmic reticulum (ER). Moreover, SE-ADM images indicated various vesicles and/or ER near the nucleus (Fig. $3 b)$. Two enlarged vesicles of red-boxed area from Fig. $3 b$ clearly reveal a spherical shape with rough surface membrane (Fig. 3c, d), and the vesicles were found to be interconnected [3].

Taken together, we developed a SE-ADM system based on SEM. This system enables high-resolution imaging of intact biological specimens in water without radiation damage. Furthermore, our system can be applied to diverse liquid samples across broad range of scientific fields, for example, nanoparticle, nanotube and organic materials [4]. 


\section{References:}

[1] T. Ogura, PLoS ONE 9 (2014), e92780.

[2] T. Ogura, Biochem. Biophys. Res. Commun. 459 (2015), p. 521.

[3] T. Okada and T. Ogura, Scientific Reports 6 (2016), p. 29169.

[4] We thank Ms. Yoko Ezaki and Miho Iida for her technical assistance. This study was supported by JSPS KAKENHI Grant-in-Aid for Scientific Research (B) (15H04365)
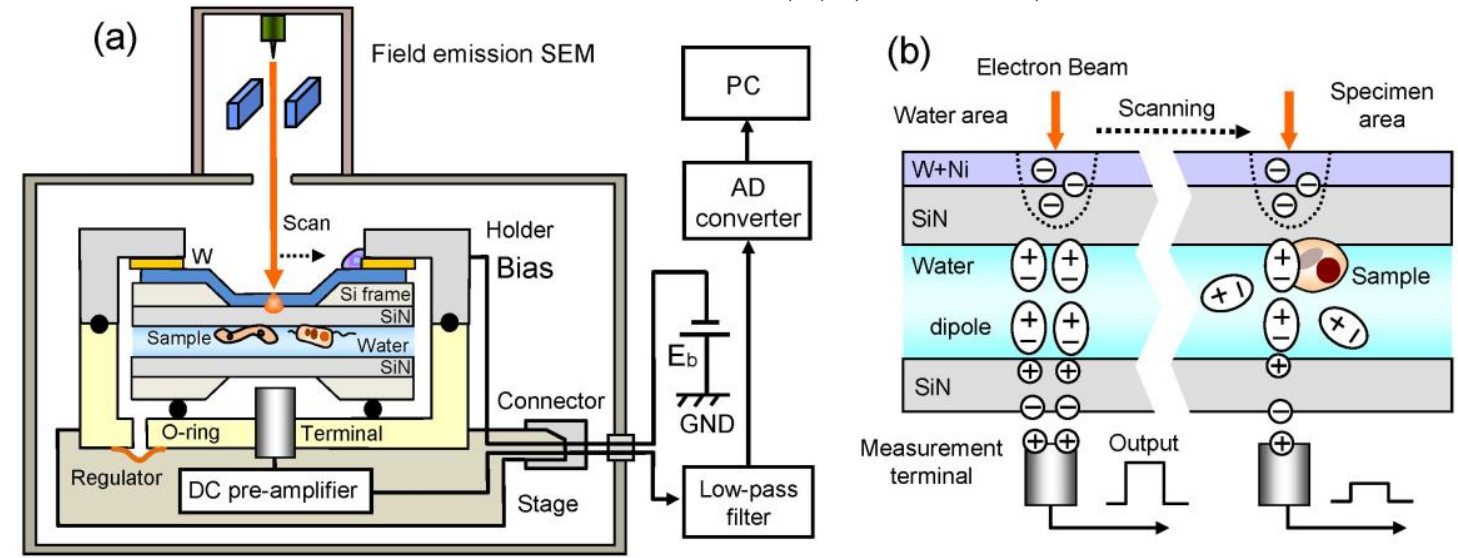

Figure 1. Experimental set-up of SE-ADM system. (a) schematic of the SE-ADM system based on field emission SEM. (b) Schematic of our hypothesis of the SE-ADM imaging method.
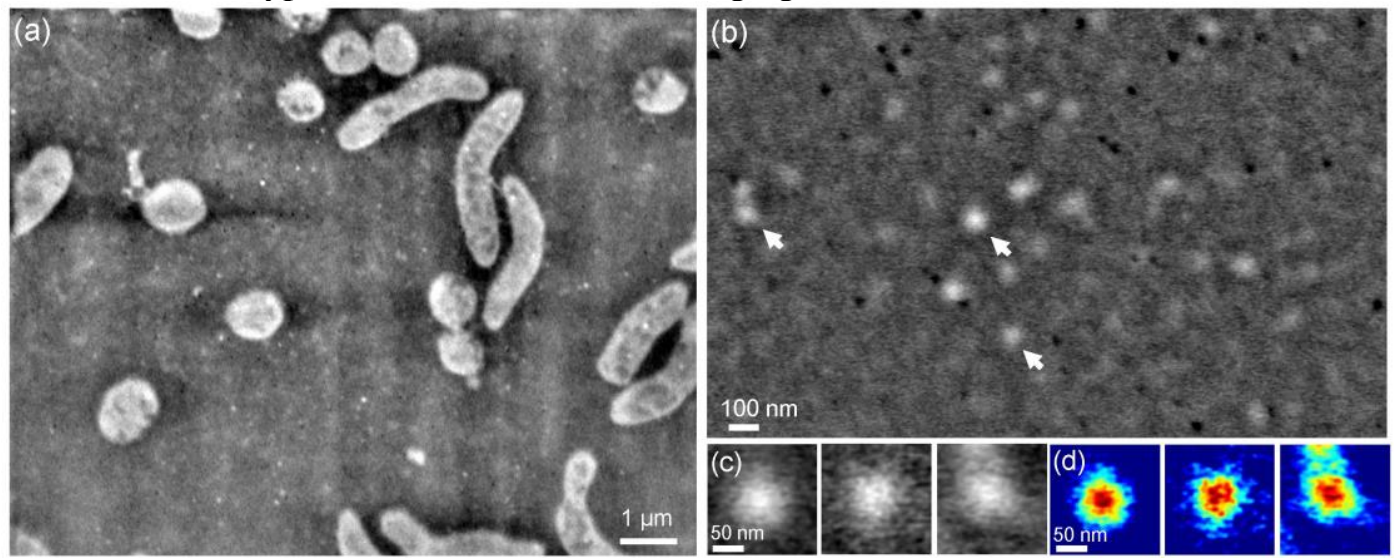

Figure 2. Imaging of intact bacteria and proteins in water using SE-ADM system. (a) The reversed contrast image of bacteria. (b) An unstained IgM particle image in water obtained by SE-ADM [2].

(c) Three individual IgM molecules indicated with white arrows. (d) Pseudo-color map of (c).
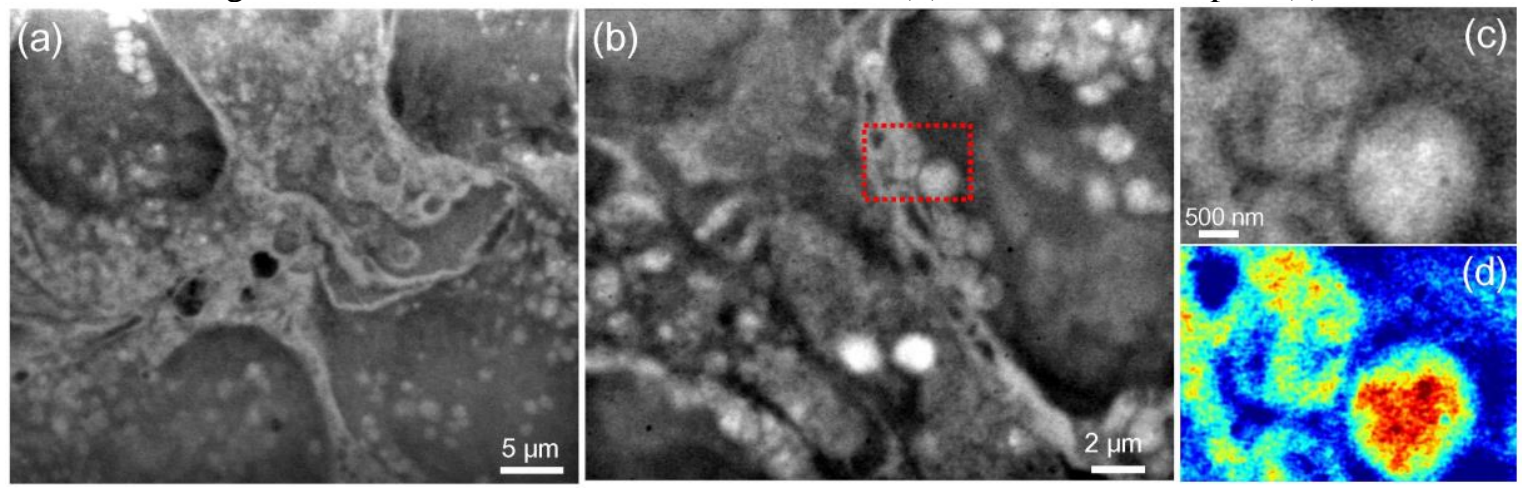

Figure 3. Observation of untreated cells in medium using SE-ADM [3]. (a) Dielectric image near the nuclear region. (b) Dielectric image of another vesicle-rich region. (c) Enlarged images of the vesicle regions highlighted in red boxes in (b). (d) Pseudo-colour maps of (c). 